\title{
Automatic Sorting of Solid Wastes Using Sensor Fusion
}

\author{
Khaled Chahine $^{\# 1}$, Bilal Ghazal ${ }^{* 2}$ \\ \# Electrical and Computer Engineering Department, ${ }^{*}$ Computer Science - Statistics Department \\ ${ }^{1}$ Beirut Arab University, Debbieh, Lebanon \\ ${ }^{2}$ Lebanese University, Zahlé, Lebanon \\ ${ }^{1}$ k.shahine@bau.edu.lb \\ 2bilal.ghazal@ul.edu.lb
}

\begin{abstract}
Nowadays solid waste is generated at an unprecedented rate due to rapid urbanization and industrialization. In the developing countries, recycling of useful materials from solid waste such as wood, plastic, glass and metal is severely constrained by limited door-to-door collection and poor means of waste sorting. Indeed, recovery of useful materials still relies on manual sorting, which is highly risky and hazardous for health. This paper details the design and implementation of an automated solid waste sorting system. The developed system is equipped with a programmable logic controller, an inductive proximity sensor, a capacitive proximity sensor, and a photoelectric sensor. Multi-sensor data fusion is used so that each material results in a different combination of sensor outputs. Once a material is identified, the controller makes a stepper motor rotate a defined angle to collect the material in its bin. Testing results show that the system is successful in sorting the four different materials.
\end{abstract}

Keywords - Waste Sorting, Recycling, Programmable Logic Controller, Automation, Sensors

\section{INTRODUCTION}

One of the most serious environmental problems facing Lebanon nowadays is the spread of different kinds of wastes due to the increase of population and lack of sustainable waste management policies. It is estimated that 5.9 million are living in Lebanon, and studies indicate that about 2.55 million tons of waste are generated in Lebanon annually. This means that each person generates 0.8 to $1.2 \mathrm{~kg}$ of waste per day, and the quantity is increasing with an annual rate of $1.65 \%$ [1].

Lebanon's garbage crisis results from many causes. First, there is a lack of long-term planning for waste management. It has been shown that $83 \%$ of the rural areas dispose of their wastes in dumps without taking any action for treating these wastes, while only $9 \%$ of urban wastes undergo sorting and recycling [2]. Also, the rapid population growth plays an important role in waste accumulation. Indeed, Lebanon's population is currently growing at around 1\% per year. Moreover, its urbanization rate is expected to reach $86 \%$ in 2020, at which point its urban population will reach 4.1 million, with a total population exceeding 6 million in the next decade [3].

Weak tax system is another aspect that leads to garbage problems. Since the growth rate is $1.65 \%$ every year, the wastes will be too much for what we can handle in Lebanon, and these taxes will be paid on how much garbage is produced by the household, such system will fund the environment programs in the country [4]. Also, the increasing number of Syrian refugees has a serious role in increasing wastes in Lebanon. According to research, municipalities are facing an increase of 30 to $40 \%$ of solid waste [5]. The municipal solid wastes produced in Lebanon are classified as follows: $52.5 \%$ are organic, $16 \%$ paper/cardboard, $11.5 \%$ plastic, 5.5\% metal, 3.5\% glass, and $11 \%$ others. These wastes are managed as follows: $15 \%$ are composted, $8 \%$ recycled, $48 \%$ landfilled and $29 \%$ openly dumped [1]. The recycling procedure starts with sorting wastes.

Materials Recovery Facilities (MRFs) sort waste by accepting the input of mixed or separated materials. One of the main problems in these facilities is the choice between manual and automated sorting techniques. Mainly, the traditional MRF is based on manual sorting when handling large volumes of materials. This method is considered very expensive and time consuming. Also, employment represents a high cost for the MRF; the annual personnel cost for five workers is approximately equivalent to the fixed asset of a million dollars' worth of capital equipment [6]. As a result, the long-run cost analysis will generally show that manual sorting is less cost efficient than the automated sorting. Manual processing can produce higher quality for recovery material; however, it is ineffective because of low sorting rates [6]. Moreover, manual sorting yields more rejected materials. For example, the workers cannot recognize a considerable portion of the HDPE and PET types of plastics. Likewise, if the worker cannot categorize the plastic resin with his naked eye, it cannot be targeted or manually sorted. It is extremely challenging for a worker to differentiate between PVC and PET types of plastics, but using automated systems these plastic resins can be separated quickly and accurately. It is therefore advantageous to have automated MRF to increase the speed of operation, reduce costs and improve quality.

In [7], a microcontroller was used as the controller of a system that sorts wastes into three categories: wet, dry, 
and metal. The main sensors used in the system were an inductive proximity sensor, resistive plates, and IR sensors. Another microcontroller-based sorting system was proposed in [8]. The system separates compost and raw wastes using sensors that detect gas level. The main drawback of microcontroller-based methods is that microcontrollers are generally not suitable for the harsh and severe operating conditions of sorting systems. These systems operate in dusty and humid environments and experience severe duty cycles, which makes microcontrollers prone to failure. Another approach to automated waste sorting is radio-frequency identification (RFID) [9]. It requires sorting at household level utilizing ordinary plastic bags, closing the bags with RFID waste identification stickers (WSI), and equipping recycling centers with RFID readers so that each bag is identified from its WSI and conveyed to its container or compactor. Although this approach of waste sorting is efficient, its accuracy depends on waste sorting at source, which cannot be guaranteed. Robotic systems have been recently employed in waste sorting. ZenRobotics Recycler is one of the examples that use robotic waste sorting. It mainly consists of a conveyor belt to deliver waste to a chamber and a robotic arm to sort waste. The robot uses several sensors such as metal detectors, cameras, and 3D laser scanners to differentiate between materials [10]. Robotic arms make the control and sorting tasks easier. In addition, they are efficient and can sort different types of waste from one stream. Such robots, however, are expensive compared to other sorting systems and difficult to maintain.

This paper details the design and implementation of a PLC-based automated solid waste sorting system able to differentiate wood, glass, plastic, and metal. Since there are no sensors dedicated to the identification of each of the four materials, fusion of the outputs of an inductive proximity sensor, a capacitive proximity sensor, and a photoelectric sensor is used so that each of the four materials results in a different combination of sensor outputs. Once a material is identified, the controller makes a stepper motor rotate a defined angle to collect the material in its bin. Testing results show that the system is successful in sorting the four different materials.

The remaining of the paper is organized as follows. Section II gives the system design. Section III describes the hardware components needed for the implementation of the design. Section IV explains how a given material affects the outputs of the installed sensors and how the resulting output combinations can be used to differentiate and sort materials. In section V, testing and results are discussed. Finally, section VI gives the summary and conclusion.

\section{SYSTEM DESIGN}

Fig. 1 shows the design of the sorting system. It consists of two parts: a part responsible for conveying objects to the sensors and another part responsible for collecting objects of the same material in the same bin. The conveyor part consists of a conveyor belt laid on a $130 \mathrm{~cm}$ by $16 \mathrm{~cm}$ conveyor track and powered by an AC motor. Holes with 2-cm inter-hole distance are drilled in the sides of the conveyor track to accommodate the following three sensors: inductive proximity sensor, capacitive proximity sensor, and photoelectric sensor. Due to the limited range of the used sensors, the conveyor track is tilted by $20^{\circ}$ so that an object slides to the side of the track where the sensors are installed. As can be seen from Fig. 1, the system is equipped with four bins since it is designed to sort four types of materials: metal, wood, glass, and plastic.

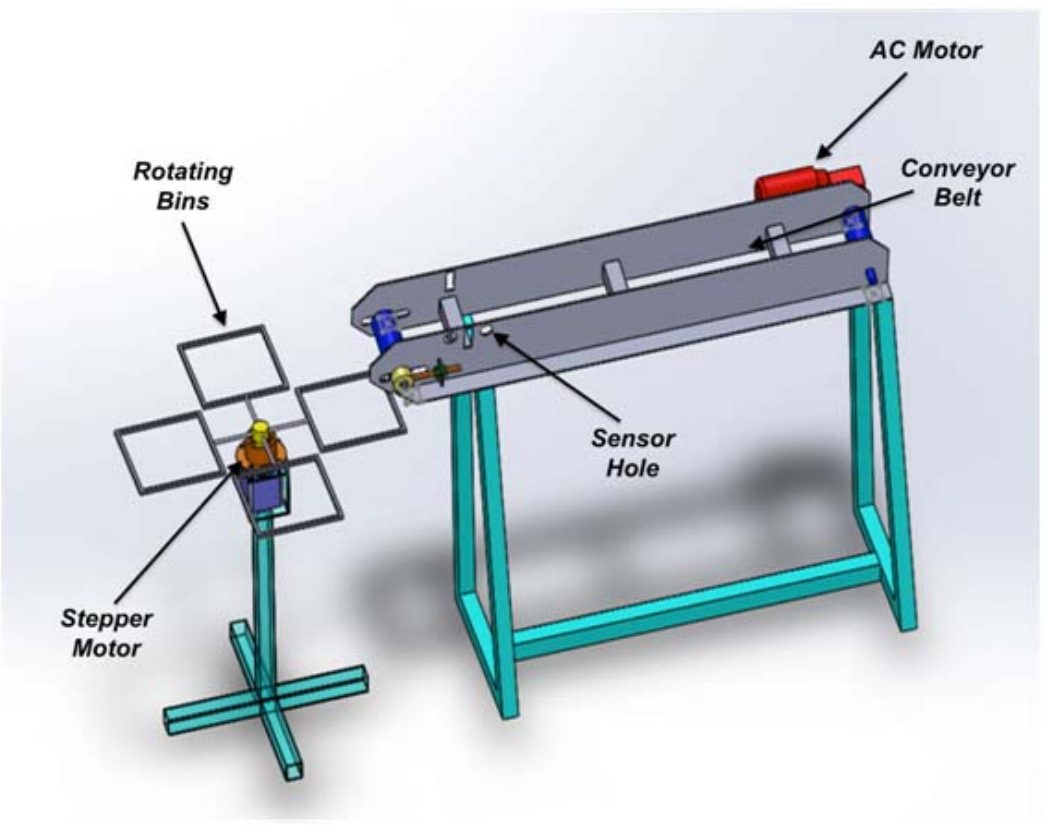

Fig. 1. The system design showing the major components of the system 
As depicted in the block diagram shown in Fig. 2, an object passing in front of the sensors results in a certain sensor output combination that enables the controller of the system to identify the material of the object. The controller then controls a stepper motor through a driver to rotate the bins till the bin corresponding to the identified material is facing the conveyor belt.

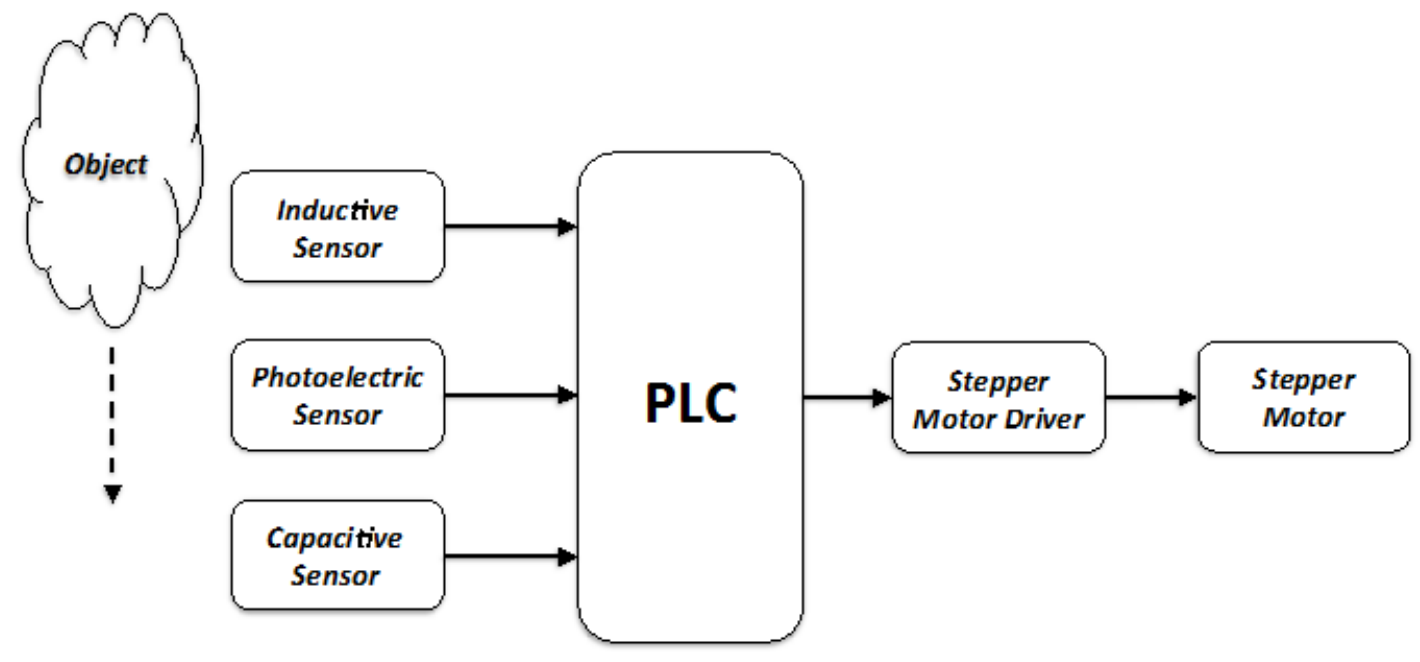

Fig.2. Block diagram of the system

III. HARDWARE COMPONENTS

This section describes the main hardware components needed for the implementation of the proposed design. These are sensors, motors, and a programmable logic controller.

\section{A. Inductive Proximity Sensor}

It is non-contact electronic proximity sensor, which is used for detecting the position of metal objects. The sensing range depends on the type of metal being tested. Ferrous metals, such as iron and steel, allow a longer sensing range, whereas nonferrous metals, like aluminum and copper, minimize the sensing range to 60\% [11]. It consists of coils and electromagnets, which are part of an LC tuned circuit that activates the oscillator. A low frequency electromagnetic field (EMF) is generated by the coils and electromagnets and radiated from the sensor's sensing surface. When a metallic object enters the EMF, the eddy current will be transmitted inside the conductive object. By absorbing the energy from EMF, this eddy current has a retroactive effect on the proximity sensor and weakens the oscillatory amplitudes. This modifies the parameters of the internal circuit thus enabling to detect any metallic approaches and to control the output of the switches. The used inductive sensor is shown in Fig. 3.

\section{B. Capacitive Proximity Sensor}

Capacitive proximity sensors, unlike the inductive ones, produce electrostatic field, which permits the sensing of metals as well as nonmetallic materials for example glass, paper, and wood. They depend on the dielectric constant of the object. Therefore, it is easier to detect materials with larger dielectric constants. Capacitive proximity sensors generate an electrostatic field and their sensing surface is formed of two metal electrodes of an unwound capacitor. When an object is near the surface, it enters the electrostatic field of these electrodes, thus the capacitance is modified in the oscillator circuit. Therefore, the oscillator starts oscillating with different amplitudes depending on the distance between the sensor and the object. When the oscillator reaches specific threshold, the output state of the sensor changes. Finally, when the object moves away, the oscillator's amplitude decreases and the sensor is switched back to its original state. The used capacitive sensor is shown in Fig. 3.

\section{Photoelectric Sensor}

Photoelectric switches detect any item through a variety of optical characteristics. Basically, they consist of a light-emitting element and a light-receiving element. When the emitted light is interrupted or reflected by the sensing object, the amount of light received is changed. The receiving element detects this change converting it to an electrical output. We have three types of optical sensors: through-beam, diffuse reflective, and retroreflective. The system uses the through-beam type shown in Fig. 3. 


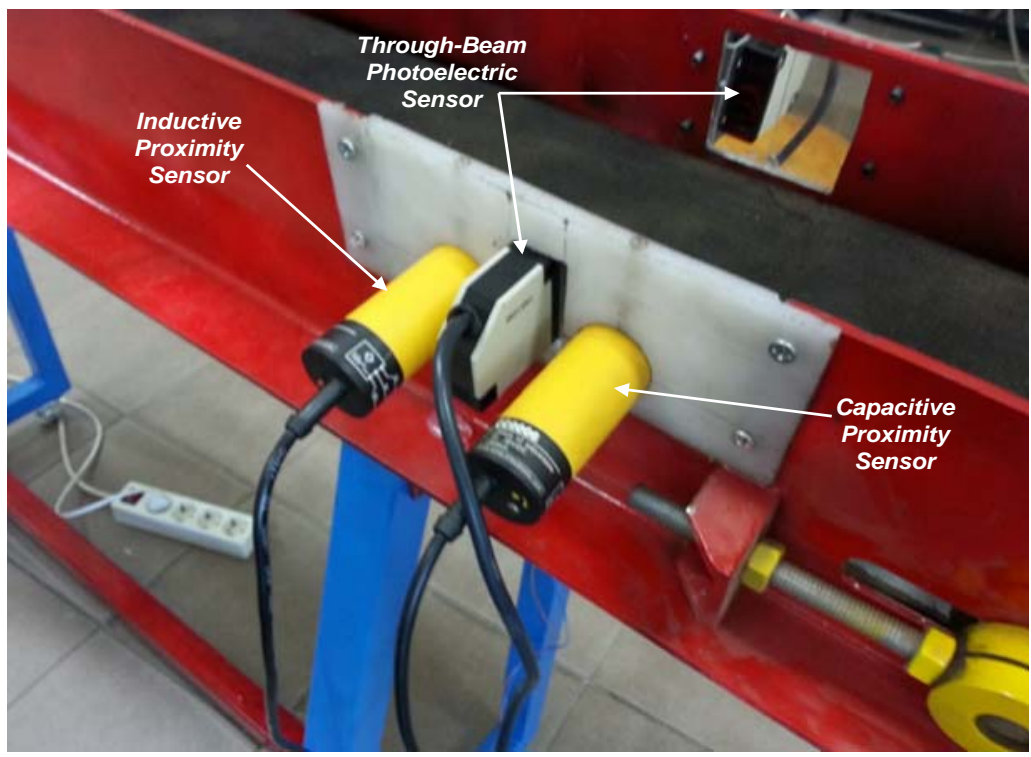

Fig.3. The inductive, photoelectric, and capacitive sensors

\section{Programmable Logic Controller}

The SIMATIC S7-1200 PLC by Siemens, shown in Fig. 4, consists of a CPU1212C that combines a microprocessor, an integrated power supply, input and output circuits, built-in PROFINET, high-speed motion control I/O and on-board analog inputs in a compact housing to create a powerful controller. The CPU contains the logical specification to monitor and manage the devices. Therefore, according to the logic of the user program, the CPU monitors the inputs and changes the outputs, which can include Boolean logic, counting, timing, complex math operations, and communications with other intelligent devices.

\section{E. AC Motor}

A $90-\mathrm{W}, 230-\mathrm{V}, 50-\mathrm{Hz}, 1350-\mathrm{rpm}$ single-phase capacitor start capacitor run induction motor is used for the conveyor belt. This motor is identical to a capacitor start motor, except that starting winding is not opened after starting. Both windings remain connected to the supply at the start, as well as for the run. A single capacitor is used for both starting and running. This improves the power factor and efficiency of the motor.

\section{F. Stepper Motor}

The $86 \mathrm{HS}$ series hybrid stepping motor with $1.8^{\circ}$ step angle is used to rotate the bins. The M542 Leadshine stepper motor driver, shown in Fig. 4, controls the stepper motor operation. There are three excitation modes for stepper motor excitation: full, half and micro step. For the system, micro-stepping mode with 1600 steps per revolution is used and the motor is connected in bipolar series.

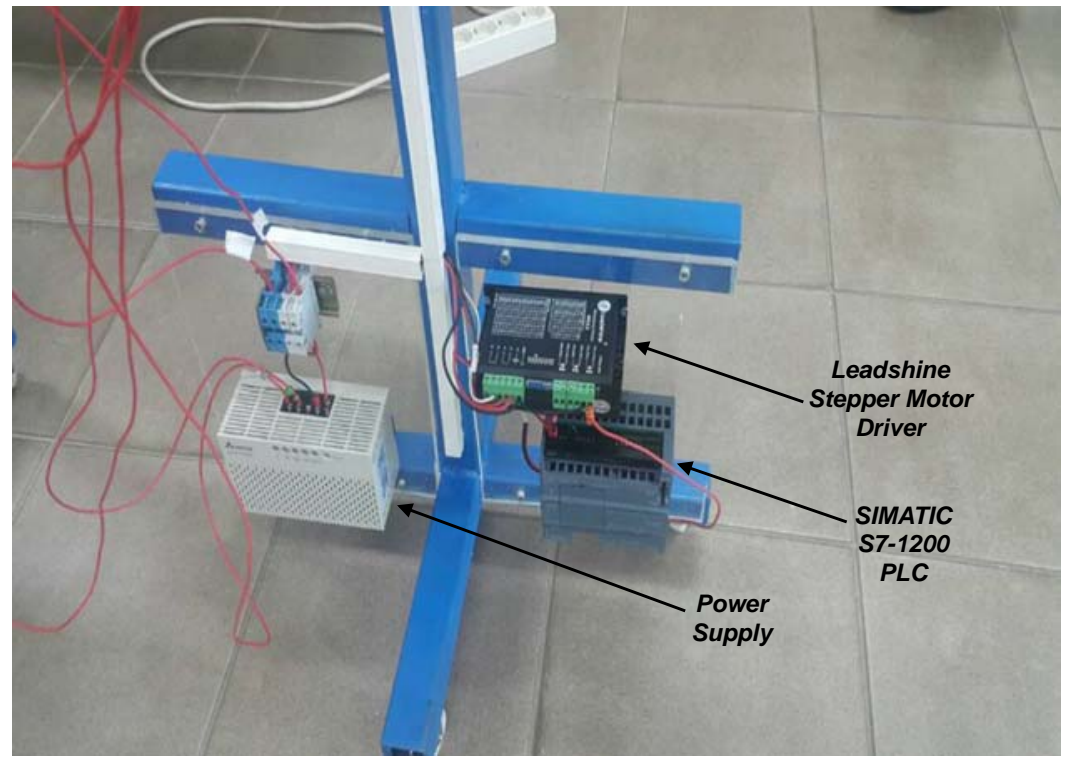

Fig.4. The power supply, SIMATIC S7-1200 PLC, and the Leadshine stepper motor driver 


\section{Sorting Mechanism}

This section explains the order in which the sensors should be installed so that each combination of sensor outputs gives one of the four materials. It also describes the operation of the complete system in a flowchart.

\section{A. Sensor Fusion}

The reason for using multi-sensor data fusion to identify a material is that, with the exception of the inductive sensor, there are no sensors dedicated to the identification of each of the four materials. Indeed, the photoelectric sensor does not detect transparent materials such as glass and plastic, but can detect opaque materials such as wood and metal, the capacitive sensor detects all materials, and the inductive sensor detects only metals. From Table I, it is evident that there are three different sensor output combinations: (S1=On, S2=On, S3=On) for metal, (S1=Off, S2=On, S3=On) for wood, and (S1=off, S2=Off, S3=On) for glass and plastic, where S1, S2, and S3 are the inductive, photoelectric, and capacitive sensors, respectively. Therefore, the system cannot distinguish glass and plastic, but only metal and wood.

TABLE I. SENSOR COMBINATIONS

\begin{tabular}{lccc}
\hline & Inductive & Photoelectric & Capacitive \\
\hline Metal & & & \\
Wood & & & \\
Glass & & & \\
Plastic & & & \\
\hline
\end{tabular}

To resolve this ambiguity, we relied on the fact that the sensitivity of the capacitive sensor varies with the material dielectric constant; the larger the dielectric constant, the longer the detection range. Plastics generally have lower dielectric constant than glass. For example, polystyrene has a dielectric constant between 2.4 and 2.6, polyethylene between 2.2 and 2.4, and polyvinyl chloride has a dielectric constant of 3.4, whereas glass has a dielectric constant between 3.7 and 10 .

Since the capacitive sensor is adjustable, it was tested to identify the range of detection beyond which it cannot detect plastic material. This range was found to be $2.2 \mathrm{~cm}$, and the sensor was adjusted to that range. As a result of this adjustment, plastics are not be detected by the capacitive sensor, and glass is detected by the capacitive sensor alone. Based on this range adjustment of the capacitive sensor, the new sensor output combinations are shown in Table II, where it can be seen that now we have four combinations instead of three: (S1=On, S2=On, S3=On) for metal, (S1=Off, S2=On, S3=On) for wood, (S1=off, S2=Off, S3=On) for glass, and (S1=off, S2=Off, S3=Off) for plastic. The ambiguity is hence resolved.

TABLE II. SENSOR COMBINATIONS AFTER ADJUSTMENT

\begin{tabular}{lccc}
\hline & Inductive & Photoelectric & Capacitive \\
\hline Metal & & C & \\
Wood & & & \\
Glass & & & \\
Plastic & & & \\
\hline
\end{tabular}

\section{B. Flowchart}

Once the system is turned on, objects move on the conveyor belt and pass in front of the three sensors in the following order: first the inductive sensor, then the photoelectric sensor and finally the capacitive sensor. Depending on the resulting combination of sensor outputs, the PLC controls the stepper motor as follows:

- $\quad$ Metal (S1=On, S2=On, S3=On): Starting from the initial position corresponding to plastic, the stepper motor rotates $90^{\circ}$, waits for $2 \mathrm{~s}$ to allow the metallic object to fall in its bin, and then rotates $270^{\circ}$ to complete one rotation and returns to its initial position.

- Wood (S1=Off, S2=On, S3=On): Starting from the initial position corresponding to plastic, the stepper motor rotates $180^{\circ}$, waits for $3 \mathrm{~s}$ to allow the wooden object to fall in its bin, and then rotates another $180^{\circ}$ to complete one rotation and returns to its initial position.

- Glass (S1=off, $\mathbf{S 2 =} \mathbf{O f f}, \mathbf{S 3}=\mathbf{O n}$ ): Starting from the initial position corresponding to plastic, the stepper motor rotates $270^{\circ}$, waits for $5 \mathrm{~s}$ to allow the glass object to fall in its bin, and then rotates $90^{\circ}$ to complete one rotation and returns to its initial position.

- $\quad$ Plastic (S1=off, S2=Off, S3=Off): The rotating bins are in their initial position corresponding to plastic and the stepper motor does not rotate. 
The reason for choosing plastic as the initial position for the rotating bins is that plastic is the most common of the four solid-waste materials. The time required for an object to move all along the conveyor belt and fall in its bin is about $16 \mathrm{~s}$, after which the system will be ready to handle another object. The flowchart of the PLC program is shown in Fig. 5.

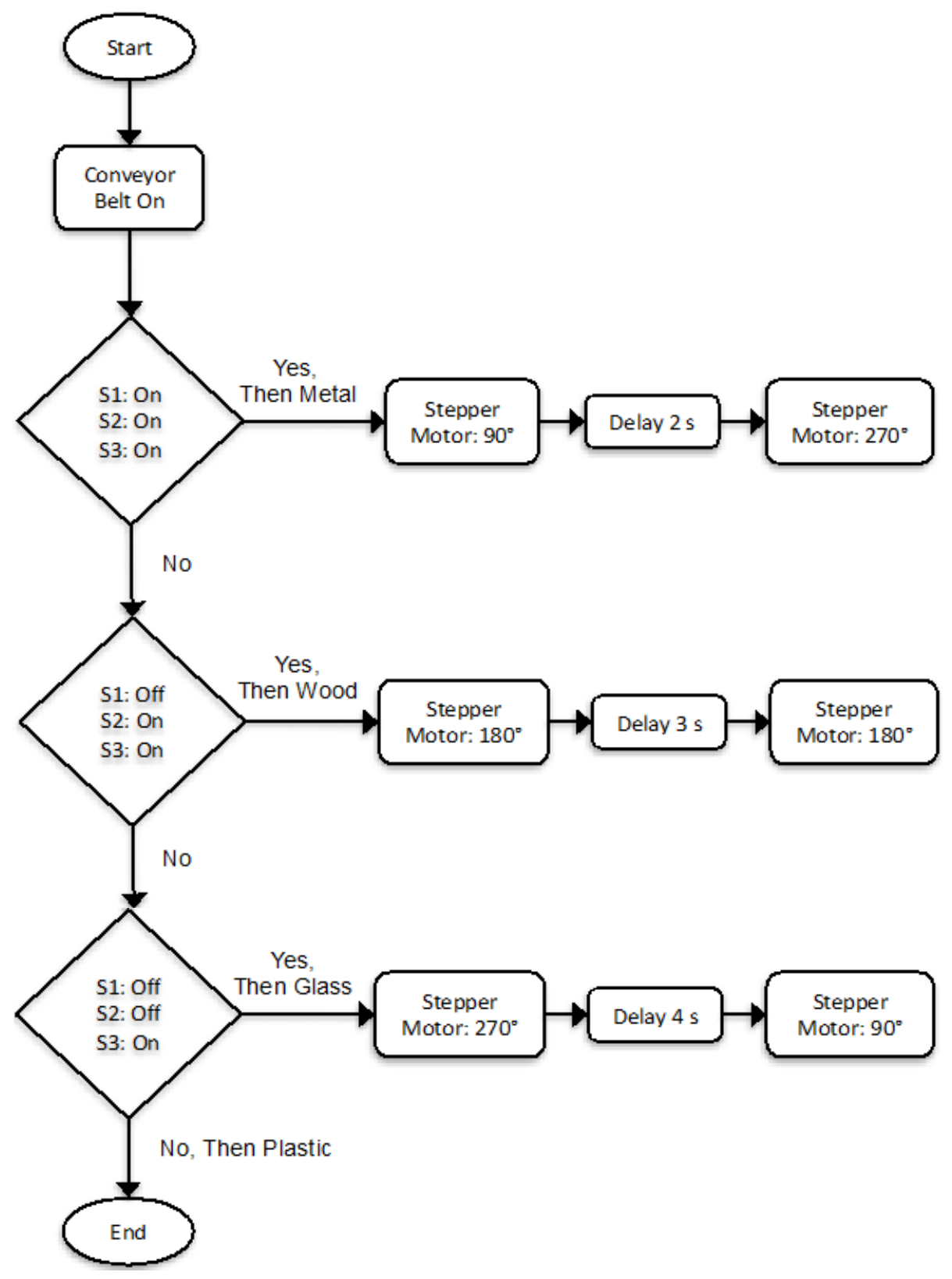

Fig.5. System flowchart

\section{Testing AND Results}

After finalizing the system's hardware and software, the system was validated through testing:

- Sensors output: Metallic, wooden, glass, and plastic objects were in turn placed on the conveyor belt in front of each of the three sensors. For all four materials, sensor outputs agreed with the results in Table II.

- $\quad$ PLC ladder diagram: For each sensor output combination, there corresponds an angle of rotation for the bins from their initial position, then a certain delay time, and finally another angle to return to initial position. The ladder diagram was validated for all four combinations through checking the stepper motor behavior against the flowchart in Fig. 5.

Testing the system repeatedly with objects of different materials and sizes showed the sorting effectiveness of the proposed design. 


\section{CONCLUSION}

This paper presented the design and implementation of an automated solid-waste sorting system capable of sorting metal, wood, glass, and plastic. The system consists of a conveying mechanism and a collection mechanism. The conveying mechanism moves objects in front of the following sensors: an inductive proximity sensor, a capacitive proximity sensor, and a photoelectric sensor. The collection mechanism rotates four bins till the bin corresponding to the identified material is facing the conveyor belt. To overcome the limitation that there are no sensors that only detect wood, glass or plastic, the system relied first on multi-sensor data fusion and second on the range adjustment of the capacitive proximity sensor so that it does not detect plastic beyond a certain range. As a result, each senor output combination identified one and only one of the four materials. Testing showed the effectiveness of the sorting system.

\section{REFERENCES}

[1] SWEEP-Net, Country Report on the Solid Waste Management in Lebanon, Sweep-Net, April 2014.

[2] J. Monzini, Municipalities and Waste Management in Lebanon, World Environment Magazine, Special Lebanon, May 2008.

[3] Lebanon Population. November 2017. Retrieved 2017-11-25, from http://worldpopulationreview.com/countries/lebanon-population/

[4] E. Fares, When Lebanon Drowns in Garbage...Again,A Separate State of Mind, A Blog by Elie Fares, July 21, (2015).Retrieved 201711-25, from https://stateofmind13.com/2015/07/21/when-lebanon-drowns-in-garbage-again/

[5] Assessment of the Impact of Syrian Refugees in Lebanon and Their Employment Profile, International Labour Organization, Regional Office for Arab States, Beirut: ILO, 2014.

[6] R. A. Dennison and J. F. Ruston, Assessing the Full Costs and Benefits of Curbside Recycling, Environmental Defense, 1995.

[7] M. K. Pushpa, A. Gupta, S. M. Shaikh, S.Jha, and V. Suchitra, Microcontroller Based Automatic Waste Segregator, IJIREEICE, May 2015.

[8] E. A. Williams and J. Bentil, Design and Implementation of a Microcontroller-Based Automatic Waste Management Sorting Unit for a Recycling Plant, AJER5(7), pp. 248 - 252, 2016.

[9] S. Dwivedi, M. Fernandes, and R. D’souza, A Review on PLC based Automatic Waste Segregator, IJARCET5 (2), 2016.

[10] T. J. Lukka, T. Tossavainen, J. V. Kujala, and Dr. T. Raiko, ZenRobotics Recycler - Robotic Sorting using Machine Learning, In Proceedings of Sensor-Based Sorting, Aachen, Germany, March 2014.

[11] F. Lamb, Components and Hardware, in Industrial Automation: Hands On, $1^{\text {st }}$ ed., McGraw-Hill Education, 2013, Ch. 3, Sec. 3.3.1, pp. 74.

\section{AUTHOR PROFILE}

Khaled Chahine received his B.E. in Electrical and Electronics Engineering from the Lebanese University, Beirut, Lebanon, in 2007, his M.S. in Electronic Systems and Electrical Engineering from Polytech'Nantes, Nantes, France, in 2007, and his Ph.D. in Electrical and Electronics Engineering from the University of Nantes, Nantes, France, in 2010. In 2010, Dr. Chahine was a postdoctoral researcher with LASMEA laboratory, Blaise Pascal University, Clermont-Ferrand, France. From 2011 to 2016, he was an assistant professor with the Department of Electrical and Electronics Engineering of the Lebanese International University, Beirut, Lebanon. Since September 2016, he has been an assistant professor with the Electrical and Computer Engineering Department of Beirut Arab University, Debbieh campus, Lebanon. His research interests include statistical signal processing, control systems, nondestructive testing and evaluation, and non-intrusive appliance load monitoring.

Bilal Ghazal received his B.E. in Electrical and Electronics Engineering in 2002 from the Lebanese University Faculty of Engineering III, Beirut, Lebanon. He received his Ph.D. degree from Claude Bernard University, Lyon France in 2009. Dr. Ghazal is currently an assistant professor in the Faculty of Sciences IV, Lebanese University, Lebanon. His research interests include microcontrollers, home automation, control systems, and image processing. 